\title{
Article
}

\section{Mechanisms of electric-field-induced alignment of block copolymer lamellae}

Pinna, Marco, Schreier, Ludwig and Zvelindovsky, Andrei V.

Available at http://clok.uclan.ac.uk/5622/

Pinna, Marco, Schreier, Ludwig and Zvelindovsky, Andrei V. ORCID: 00000003-4464-3254 (2009) Mechanisms of electric-field-induced alignment of block copolymer lamellae. Soft Matter, 5 (5). pp. 970-973. ISSN 1744-683X

It is advisable to refer to the publisher's version if you intend to cite from the work. http://dx.doi.org/10.1039/B814969K

For more information about UCLan's research in this area go to http://www.uclan.ac.uk/researchgroups/ and search for < name of research Group>.

For information about Research generally at UCLan please go to http://www.uclan.ac.uk/research/

All outputs in CLoK are protected by Intellectual Property Rights law, including Copyright law. Copyright, IPR and Moral Rights for the works on this site are retained by the individual authors and/or other copyright owners. Terms and conditions for use of this material are defined in the policies page.

\section{CLoK}

Central Lancashire online Knowledge www.clok.uclan.ac.uk 


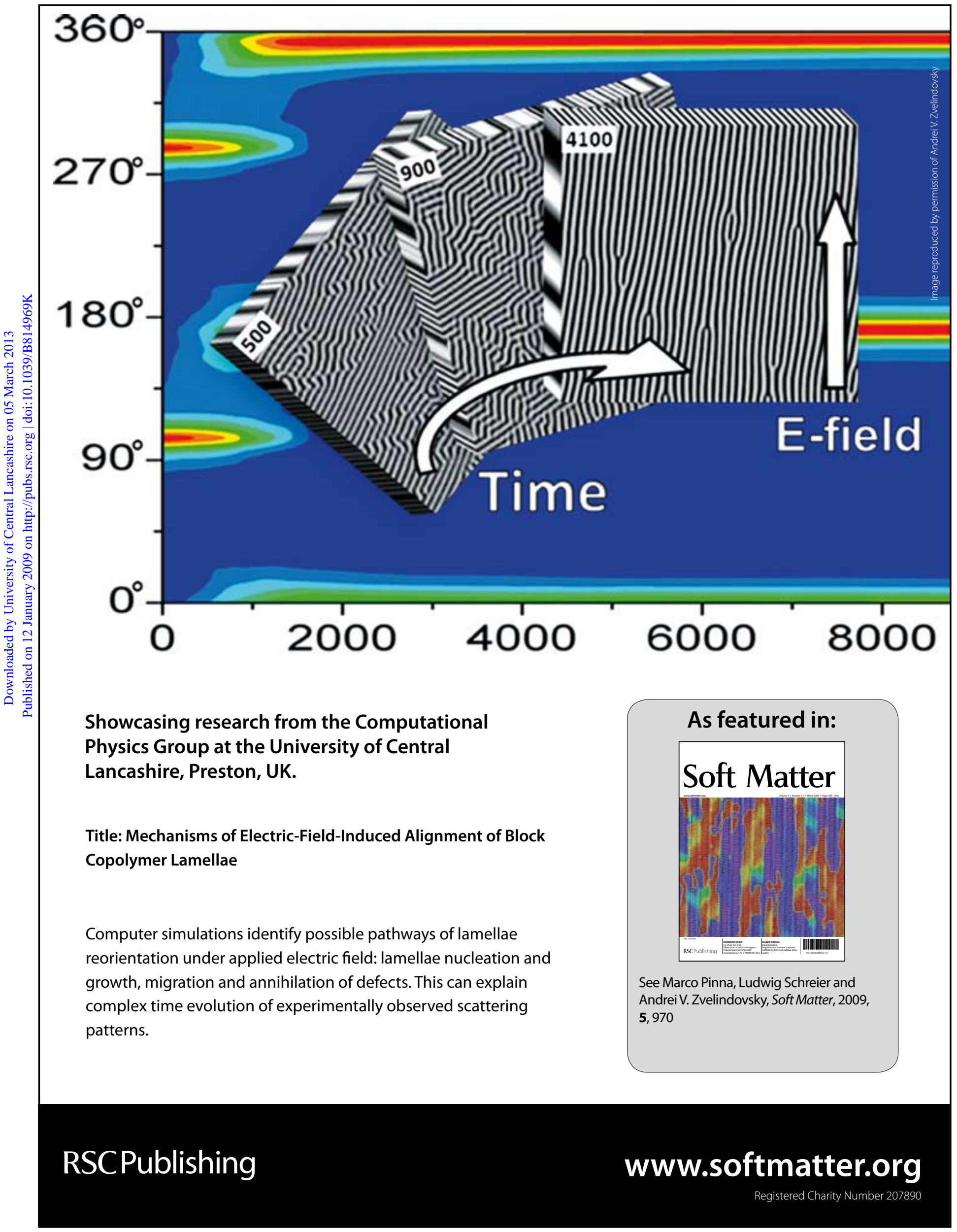




\title{
Mechanisms of electric-field-induced alignment of block copolymer lamellae
}

\author{
Marco Pinna, Ludwig Schreier† and Andrei V. Zvelindovsky* \\ Received 27th August 2008, Accepted 12th November 2008 \\ First published as an Advance Article on the web 12th January 2009 \\ DOI: $10.1039 / b 814969 k$
}

We demonstrate that two mechanisms of lamellae reorientation observed experimentally under applied electric field [A. Böker H. Elbs, H. Hänsel, A. Knoll, S. Ludwigs, H. Zettl, V. Urban, V. Abetz, A. H. E. Müller and G. Krausch, Phys. Rev. Lett., 2002, 89, 135502] which have been previously described within dynamic self consistent field theory [A. V. Zvelindovsky and G. J. A. Sevink, Phys. Rev. Lett., 2003, 90, 049601] can be fully explained within a much more simple model using the Ginzburg-Landau Hamiltonian. A third alignment mechanism has been identified which was not previously reported. A more complete picture of reorientation under electric field emerges that clarifies the crucial role of structural defects.

\section{Introduction}

Böker et al. ${ }^{1}$ identified experimentally two distinct microscopic mechanisms of electric-field-induced alignment of diblock copolymer microdomains. Close to order-disorder transition (ODT) the scattering peak from lamellae parallel to the electrodes quickly disappears and a new peak from the lamellae parallel to the electric field appears. Further from ODT the peak merely rotates from the initial position towards the final one. This observation can be attributed to nucleation and rotation. ${ }^{1}$

In the subsequent comment ${ }^{2}$ we showed that the experimental behaviour can be explained in terms of dynamic self consistent field theory (SCFT) for ideal Gaussian chains with mean field interactions. Two microscopic mechanisms were identified, (see Fig. 1). SCFT is based on the polymer path integral calculation and is computationally very expensive. We argued before that SCFT is better suited to describe kinetics of specific chemical systems with various metastable transient states compared to theories based on the phenomenal free energies. ${ }^{3}$ This statement,

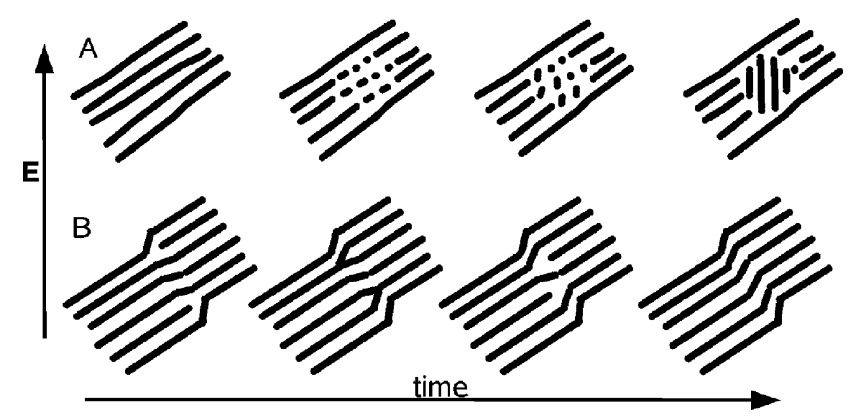

Fig. 1 A schematic representation of lamellar microdomains reorientation under an electric field E: (A) nucleation and growth and (B) rotation via defect annihilation.

Computational Physics Group, School of Computing Engineering and Physical Sciences, University of Central Lancashire, Preston, United Kingdom PR1 2HE.E-mail: AVZvelindovsky@uclan.ac.uk

$\dagger$ Present address: Department of Physics, University of Applied Sciences, Wiesbaden, Germany however, was never rigorously verified. From the physics point of view a minimal model based on phenomenal free energy provides a clearer understanding and a transparent link to other fields of physics. In the present work we show that at least the experimental behaviour of lamellae under an electric field ${ }^{1}$ does not require cumbersome SCFT and can be fully explained with a much more simple model within the spirit of the GinzburgLandau approach. To prove this statement we perform simulations of symmetric diblock copolymer melt in two dimensions, which is the same setup as we used in our previous SCFT calculation. ${ }^{2}$ Moreover, we found an additional phase transition scenario not observed in our previous SCFT calculation, ${ }^{2}$ which makes the whole picture more complete. As a consequence, the Ginzburg-Landau approach and SCFT can be used in future in tandem, where the Ginzburg-Landau approach serves as precursor to SCFT. The situation is not unique to the electric field problem. Very recently, the Landau-Ginzburg approach was used to study block copolymer vesicle formation. ${ }^{4}$ The kinetic pathway, when a bilayer platelet bends to form a spherical vesicle, is found to be topologically the same as one reported earlier by dynamic SCFT calculation. ${ }^{5}$

Block copolymers are a prominent example of nanostructured materials as they form a large variety of well ordered microdomain structures of macromolecular dimensions. Electric field manipulation of these structures is receiving large attention (see ref. 6 and 7 for comprehensive reviews on the subject). Here, we address the simplest structure which is a sandwich-like or a lamellar structure formed by a symmetric diblock copolymer. We are interested in full kinetic pathways of reorientation while other theoretical groups were aiming for stability analysis of modes in lamellae under electric field using either the GinzburgLandau approach or SCFT.8,9

\section{Method}

We implement a Ginzburg-Landau approach in a cell dynamics simulation (CDS), which is a very fast computational technique $^{10-18}$ and can serve as a research precursor for more elaborate but slow methods like SCFT. For a symmetric AB diblock copolymer the structure can be described by an order parameter 
$\psi(\mathbf{r}, t)=\phi_{\mathrm{A}}-\phi_{\mathrm{B}}$, where $\phi_{\mathrm{A}}$ and $\phi_{\mathrm{B}}$ are the local volume fractions of $\mathrm{A}$ and $\mathrm{B}$ monomers respectively. The time evolution of the order parameter is given by the Cahn-Hilliard-Cook (or the time-dependent Ginzburg-Landau) equation, ${ }^{10-18}$ which in the presence of an applied electric field (along the $y$ direction) becomes: $:^{2,15-17,19}$

$$
\frac{\partial \psi}{\partial t}=M \nabla^{2}\left(\frac{\delta F[\psi]}{\delta \psi}\right)+\tilde{\alpha} \nabla_{y}^{2} \psi
$$

where $M$ is a phenomenal mobility constant and

$$
\tilde{\alpha}=\frac{\left(\varepsilon_{\mathrm{A}}-\varepsilon_{\mathrm{B}}\right)^{2} E_{0}^{2} \nu}{2 \pi\left(\varepsilon_{\mathrm{A}}+\varepsilon_{\mathrm{B}}\right) k T}
$$

Here $\varepsilon_{\mathrm{A}}$ and $\varepsilon_{\mathrm{B}}$ are the dielectric constants of the A and B block respectively, $E_{0}$ is the strength of the electric field and $\nu$ is the volume of one polymer chain. ${ }^{17,19}$ The last term in eqn 1 was derived using the expression for the chemical potential $\mu=\mu^{0}-$ $(\partial \varepsilon / \partial \psi)_{T} E^{2} / 8 \pi,{ }^{20}$ and expanding the dielectric constant $\varepsilon$ and Maxwell equation $\operatorname{div}(\varepsilon \boldsymbol{E})=0$ in the leading power of $\psi$. The dimensionless time is $t M / a_{0}{ }^{2}$, where the lattice cell size $a_{0}$ and $M$ are set to 1 . The free energy function is: ${ }^{11-15}$

$$
\begin{aligned}
F[\psi] & =\int d \mathbf{r}\left[H(\psi)+\frac{D}{2}|\nabla \psi|^{2}\right] \\
& +\frac{B}{2} \int d \mathbf{r} \int d \mathbf{r}^{\prime} G\left(\mathbf{r}-\mathbf{r}^{\prime}\right) \psi(\mathbf{r}) \psi\left(\mathbf{r}^{\prime}\right)
\end{aligned}
$$

where

$$
H(\psi)=-\frac{\tau}{2} \psi^{2}+\frac{u}{4} \psi^{4}
$$

with $\tau, u, D, B$ being phenomenal parameters, and $G\left(\mathbf{r}-\mathbf{r}^{\prime}\right)$ is the Laplace equation Green function.

All these parameters can be related to molecular characteristics. According to Ohta and Kawasaki $\tau, D$, and $B$ can be expressed in terms of degree of polymerisation $N$, the segment length $b$ and the Flory-Huggins parameter $\chi$ (which is inversely proportional to temperature) as: ${ }^{18,21} \tau=\frac{1}{N}(2 N \chi-3.6), D=$ $b^{2} / 12, B=36 / N^{2} b^{2}$. In simulation we use the dimensionless parameters $\tilde{D}=D / a_{0}{ }^{2}$ and $\tilde{B}=B a_{0}{ }^{2}$ (for simplicity we keep the notations $D$ and $B$ instead of $\tilde{D}$ and $\tilde{B}$ ). The parameter $u$ does not allow for a compact representation and can be computed by evaluating the appropriate vertex function given by Leibler. $^{22}$ It is a very complex function which can be only approximately replaced by a constant. We believe that the phenomenon we are studying is quite general and, hence, we allow the freedom of choosing the parameters in eqn 3 and 4 as phenomenal constants not specifically limited to diblock copolymers.

\section{Results and discussion}

All simulations were performed using periodic boundary conditions in a two dimensional (2D) box of $300 \times 300$ grid points (in units of $a_{0}$ ). The parameters used in simulations are: $f=0.5, u=$ $0.5, D=0.5$ and $B=0.02 .{ }^{13}$ The simulation results for different temperature parameter $\tau$ and electric field parameter $\tilde{\alpha}$ are shown in Fig. 2. Initial configuration was created by using a shear flow

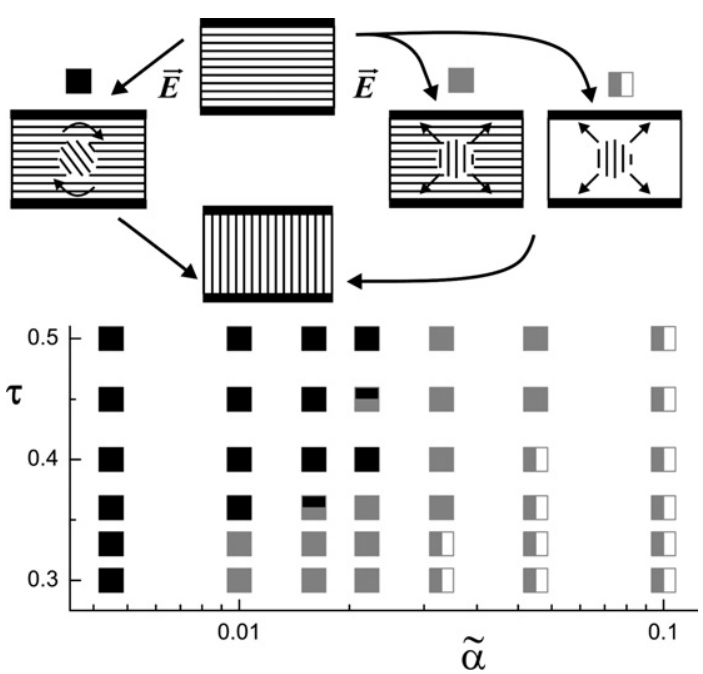

Fig. 2 Top: a schematic representation of lamellar domains reorientation between two electrodes via different mechanisms: rotation (black boxes), nucleation and growth with and without melting (grey/ white and grey boxes correspondingly). Modified (with permission) from ref. 7. Bottom: a simulation diagram of the mechanisms. Box shades correspond to the mechanisms in the Top section. Black/gray boxes indicate a mixture of rotation and nucleation without melting. The parameters on the axes are $\tilde{\alpha} \propto E_{0}{ }^{2}$ (eqn 2) and $\tau \propto \chi$, where $\chi$ is the Flory-Huggins parameter.

along the $x$-direction for every value of $\tau$, and then an electric field along the $y$-direction was applied. In all simulations the initial configurations are similar to the one shown in Fig. 3, $t=0$, and have many defects.
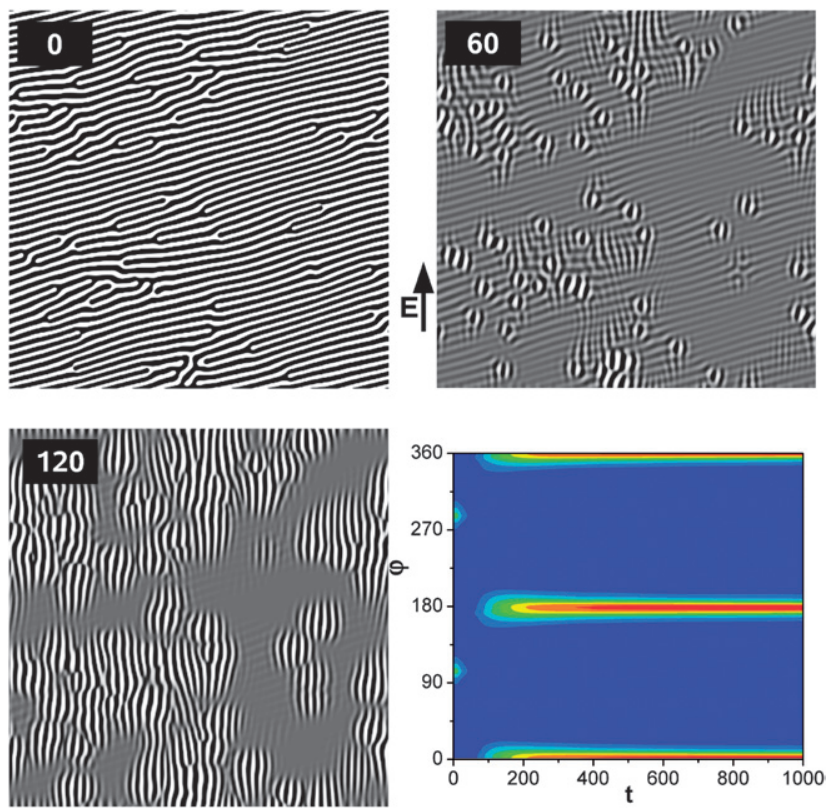

Fig. 3 Nucleation and growth with partial melting (half-grey boxes in Fig. 2) for $\tau=0.36$ and $\tilde{\alpha}=0.1$. The direction of the electric field is indicated by the arrow. The electric field was switched on at $t=0$ (elapsed time $t$ is shown in black rectangles). Time development of the scattering intensity as a function of the azimuthal angle $\varphi$ (compare with ref. 1) in the presence of the electric field is shown in the right bottom image. 
Both mechanisms depicted in Fig. 1 are observed. Our simulations show that the alignment mechanisms are discriminated by energetics, namely, by the balance of the ponderomotive force ${ }^{20}$ (controlled by $\tilde{\alpha}$ ) and the strength of the interface (controlled by $\tau)$. For weaker electric fields (parameter $\tilde{\alpha}$ ) and further away from ODT (low temperature, parameter $\tau$ ) rotation mechanism is observed. At stronger fields and/or higher temperatures, nucleation and growth mechanism occurs. We distinguish two types of this mechanism.

At higher temperatures the segregation of copolymer blocks is weaker and the interface between blocks is more diffuse. Such interface cannot withstand the electric field, thus undulation and peristaltic modes grow in lamellae (Fig. 3, $t=60$ ). The lamellae partially melt. Some of the transient structure (Fig. 3, $t=60$ ) is similar to the mixed morphology, which is a superposition of parallel and perpendicular lamellae to the electric field, found by the static analysis in ref. 23 [Fig. 4(b) therein]. Fig. 3 at $t=$ 0 shows the starting configuration with many defects mostly in the form of dislocations. When the electric field is applied these defects serve as nuclei of new lamellar phase in the direction of the electric field (Fig. 3, $t=60$ ). These nuclei form small clusters which grow and eventually merge (compare Fig. 3 at $t=60$ and at $t=120$ ). This mechanism can be also deducted from the behaviour of scattering function which is calculated as the Fourier transform squared of the real space images (Fig. 3, bottom-right). We observe a destruction of the initial peaks followed by a buildup of scattering at the final peak position. The peaks are separated by approximately 90 degrees with no intermediate orientations observed (Fig. 3). Partial melting manifests itself in a drastic drop of intensity between $t=30$ and $t=70$. This sub-type of nucleation and growth mechanism was not reported in ref. 2 due to the much narrower parameter range studied by SCFT. The possibility of a melting mechanism was speculated about in ref. 24. However, that was done based on static considerations and the conclusion was not very definite. Experimentally this mechanism was indicated only most recently in the trial in situ AFM measurements by Böker. ${ }^{25}$ Partial melting is not unique to our model. It was also found by dynamic SCFT calculation as an intermediate phase in the case of an electric field applied to a spherical morphology close to ODT phase boundary. ${ }^{19}$ It was also reported as electric field induced remixing for polymer solutions within phenomenal mean-field theory (static analysis). ${ }^{26}$ It was also predicted for block copolymer melts as a result of suppression of the composition fluctuations by electric field (static treatment), ${ }^{27}$ which we do not consider here.

Simulation also reveals details of the nucleation and growth mechanism without partial melting (Fig. 4), which is observed at lower temperatures (higher $\tau$ ). Instead of melting, lamellae break into short micelles (see Fig. 1A) which recombine into clusters of lamellae parallel to the electric field. This process starts around defects (Fig. 4, $t=500$ ). The clusters grow via the grain boundary migration (Fig. 4, $t=900$ ). This mechanism is also seen in the scattering function evolution, which shows initial and final peaks shifted by approximately 90 degrees (Fig. 4). There is no drop in the overall intensity as the peaks overlap in the region between $t=190$ and $t=1600$. Therefore, the structure does not melt. We found this type of nucleation and grow mechanism earlier with SCFT calculations in..$^{2,19,28-30}$ When the temperature is even lower
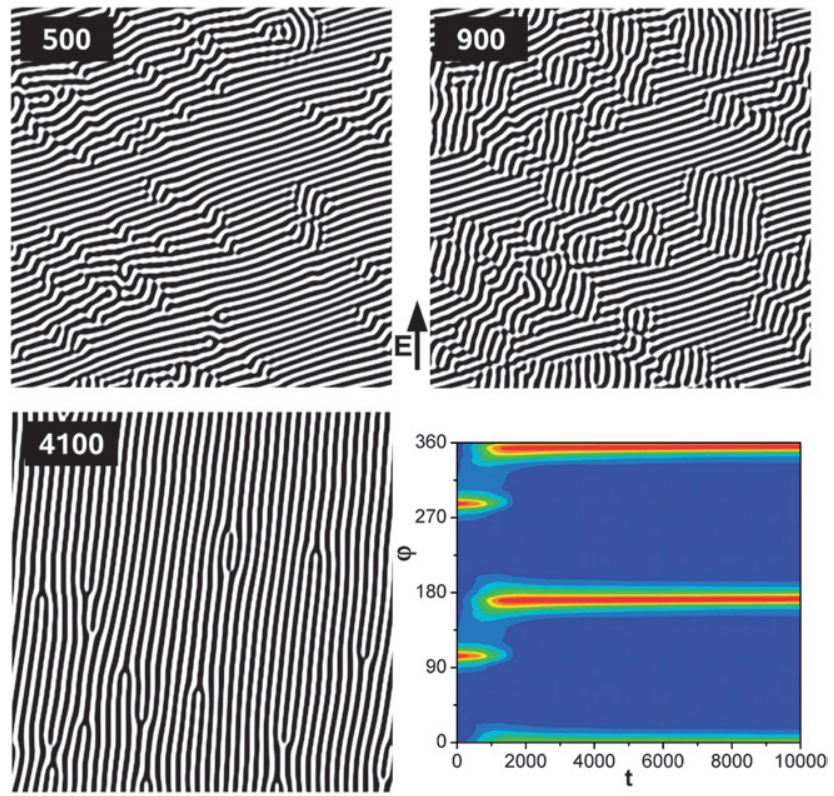

Fig. 4 Nucleation and growth without partial melting (grey boxes in Fig. 2) for $\tau=0.36$ and $\tilde{\alpha}=0.02$. The layout of the figure is the same as Fig. 3.

the segregation between blocks is stronger and the interfaces between them are sharper. Undulation and peristaltic instability modes are suppressed (compare Fig. 5 with 3 and 4). In such a case the only possible movement is defect migration (see Fig. 1B). Two dislocations with different orientation in space move in the opposite directions perpendicular to lamellar stripes. When they meet they both annihilate resulting in a local lamellae
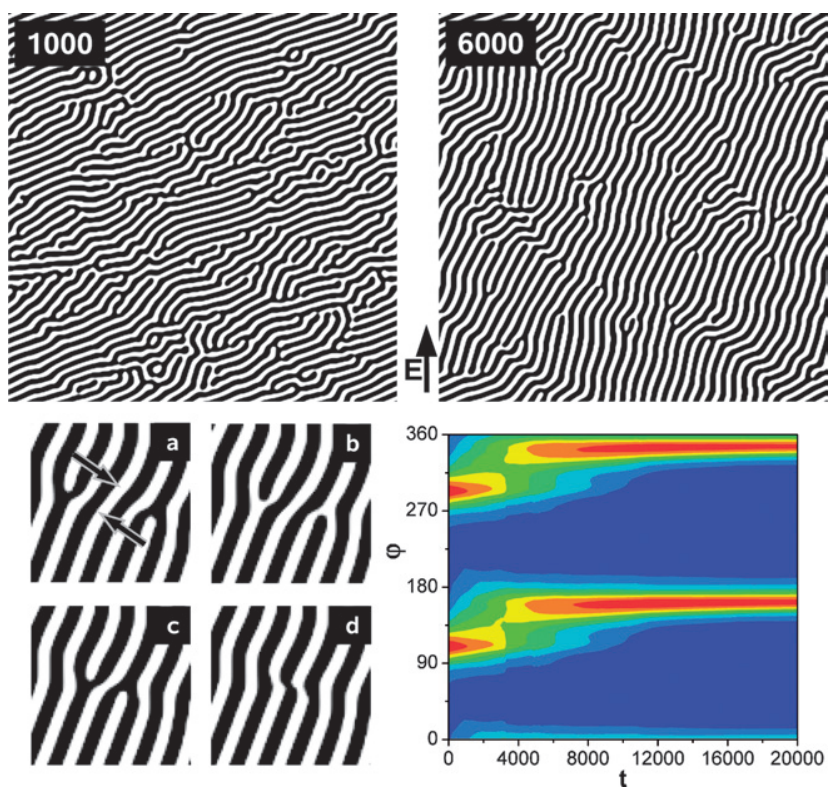

Fig. 5 Rotation (black boxes in Fig. 2) for $\tau=0.40$ and $\tilde{\alpha}=0.015$. The layout of the figure is similar to Fig. 3 apart from the bottom left panel which shows crops at times $t$ : 9600 (a), 10100 (b), 10500 (c), 10700 (d). Directions of defect movement are shown by the two arrows in part (a). 
rotation (Fig. 5a-d). After many annihilation events the overall pattern rotates Fig. 5, $t=6000$, which is also seen in a gradual shift of scattering intensity peaks (Fig. 5). Such scattering behaviour was observed experimentally and initially was interpreted as whole grain rotation (see schematic representation in Fig. 2, top-left cartoon) due to change in kinetics. ${ }^{1}$ Our interpretation (individual defect movements) is different and is due to the balance of energetic contributions. Our system stops rotating when all defects in the simulation box annihilate, the same behaviour was found by dynamic SCFT calculation in ref. 2. Confirmation of this mechanism by real space experiments is still awaited.

The observed defect movement perpendicular to lamellae is not unique for our system: it was also found in other systems forming stripes patterns. For instance, it was experimentally observed and theoretically described for electroconvection patterns, which are governed by different physics. $^{31}$

The boundary between the two alignment mechanisms is not sharp and they can coexist which is indicated by the black/grey boxes in Fig. 2. Individual defect movement is present to some extent in systems undergoing nucleation and growth reorientation and can be seen in the light blue shift in the scattering function in Fig. 4 between $t=500$ and $t=2000$. Defect movement is dominant in the same system at latest stages of reorientation (Fig. 4, $t=4100$ ). This is due to the fact that at this stage the angle between lamellae and the electric field is small and as a result the ponderomotive force is too weak to cause any instability. ${ }^{8}$ Therefore, no new nucleus can form and grow.

\section{Conclusion}

In conclusion kinetic mechanisms of microdomain orientation by electric DC field of diblock copolymer systems have been investigated within the most simple physical model based on the Landau Hamiltonian. Microdomain rotation and nucleation with and without partial melting are found to be controlled by the interplay between strength of the electric field and degree of microphase separation (vicinity to ODT). Structural defects are essential in realisation of either of these mechanisms.

\section{Acknowledgements}

This work is supported by Accelrys Ltd via EPSRC CASE research studentship. All simulations were performed on SGI Altix 3700 supercomputer at UCLan High Performance Computing Facilities. LS acknowledges an undergraduate research grant from LDU, UCLan, which enabled him to visit Preston. AVZ is thankful to the late Professor Lorenz Kramer, fruitful discussions with whom in Bayreuth, in particular on the topic of defects movement, inspired the present work.

\section{References}

1 A. Böker, H. Elbs, H. Hänsel, A. Knoll, S. Ludwigs, H. Zettl, V. Urban, V. Abetz, A. H. E. Müller and G. Krausch, Phys. Rev. Lett., 2002, 89, 135502 .

2 A. V. Zvelindovsky and G. J. A. Sevink, Phys. Rev. Lett., 2003, 90, 049601 .

3 B. A. C. van Vlimmeren, N. M. Maurits, A. V. Zvelindovsky, G. J. A. Sevink and J. G. E. M. Fraaije, Macromolecules, 1999, 32, 646

4 T. Uneyama, J. Chem. Phys., 2007, 126, 114902.

5 G. J. A. Sevink and A. V. Zvelindovsky, Macromolecules, 2005, 38, 7502.

6 T. Xu, J. Wang, and T. P. Russell, in Nanostructured Soft Matter, ed. A. V. Zvelindovsky, Springer, Dordrecht, 2007, (part 1), ch. 6.

7 A. Böker, in Nanostructured Soft Matter, ed. A. V. Zvelindovsky, Springer, Dordrecht, 2007, (part 1), ch. 7.

8 A. Onuki and J. Fukuda, Macromolecules, 1995, 28, 8788.

9 M. W. Matsen, Soft Matter, 2006, 2, 1048.

10 Y. Oono and S. Puri, Phys. Rev. A, 1988, 38, 434.

11 M. Bahiana and Y. Oono, Phys. Rev. A, 1990, 41, 6763.

12 S. Qi and Z. G. Wang, Phys. Rev. E, 1997, 55, 1682.

13 S. R. Ren, I. W. Hamley, G. J. A. Sevink, A. V. Zvelindovsky and J. G. E. M. Fraaije, Macromol. Theory Simul., 2002, 11, 123.

14 S. R. Ren and I. W. Hamley, Macromolecules, 2001, 34, 116.

15 M. Pinna, A. V. Zvelindovsky, S. Todd and G. Goldbeck-Wood, J. Chem. Phys., 2006, 125, 154905.

16 X. Guo, M. Pinna and A. V. Zvelindovsky, Macromol. Theory Simul., $2007,16,779$.

17 M. Pinna and A. V. Zvelindovsky, Soft Matter, 2008, 4, 316.

18 M. Pinna, X. Guo and A. V. Zvelindovsky, Polymer, 2008, 49, 2797.

19 K. S. Lyakhova, A. V. Zvelindovsky and G. J. A. Sevink, Macromolecules, 2006, 39, 3024.

20 L. D. Landau and E. M. Lifshitz, Electrodynamics of Continuous Media, Pergamon, Oxford, 1960, ch. 2.

21 T. Ohta and K. Kawasaki, Macromolecules, 1986, 19, 2621.

22 L. Leibler, Macromolecules, 1980, 13, 1602.

23 Y. Tsori and D. Andelman, Macromolecules, 2002, 35, 5161.

24 K. Amundson, E. Helfand, X. Quan, S. D. Hudson and S. D. Smith, Macromolecules, 1994, 27, 6559.

25 A. Böker, private communication.

26 D. Wirtz, K. Berend and G. G. Fuller, Macromolecules, 1992, 25, 7234.

27 I. Gunkel, S. Stepanow, T. Thurn-Albrecht and S. Trimper, Macromolecules, 2007, 40, 2186.

28 A. Böker, H. Elbs, H. Hänsel, A. Knoll, S. Ludwigs, H. Zettl, A. V. Zvelindovsky, G. J. A. Sevink, V. Urban, V. Abetz, A. H. E. Müller and G. Krausch, Macromolecules, 2003, 36, 8078.

29 K. Schmidt, A. Böker, H. Zettl, F. Schubert, H. Hänsel, F. Fischer, T. M. Weiss, V. Abetz, A. V. Zvelindovsky, G. J. A. Sevink and G. Krausch, Langmuir, 2005, 21, 11974.

30 K. Schmidt, H. G. Schoberth, F. Schubert, H. Hänsel, F. Fischer, T. M. Weiss, G. J. A. Sevink, A. V. Zvelindovsky, A. Böker and G. Krausch, Soft Matter, 2007, 3, 448.

31 P. Tóth, N. Éber, T. M. Bock, A. Buka and L. Kramer, Europhys. Lett., 2002, 57, 824. 